55.

\section{Analysis of Calls Under-triaged by Priority Medical Dispatch in San Diego}

W. Eric Strukel, BS, James V. Dunford, MD

Department of Emergency Medicine, University of CaliforniaSan Diego Medical Center, San Diego, California USA

Purpose: To identify calls "under-triaged" by priority medical dispatch and determine causes and pre-hospital outcomes.

Methods: 6 month retrospective analysis identified calls dispatched "low priority" to which medics assigned "high acuity" transports (acute status or requiring ALS meds; not just IV/O2/monitor). CAD data, paramedic run-sheets, and audiotapes were reviewed to determine optimal dispatch levels and transport codes. "Under-triage" was defined as calls warranting "high priority" dispatch based on evidence from the run-sheet. Dispatcher, calling party, and patient data influencing "undertriage" were assessed.

Results: In 1995, 11,178/70,887 (16\%) medical aid requests were dispatched "low priority". 201(1.8\%) were subsequently assigned "high acuity" transport codes by paramedics. $105 / 5,737$ such consecutive patients were analyzed from July-December 1995. 6 were excluded due to incomplete data. After review, 42/99 actually warranted "high priority" dispatch. 7 had potential life/limb threatening injuries; 35 required ALS intervention. None had adverse prehospital outcome. Undertriage was associated with dispatcher error, information relayed from law enforcement officers ( $O R=3.4$, CI: $1.2-10)$ calls involving alcohol $(\mathrm{OR}=2.8, \mathrm{CI}: 0.9-9.2)$ or patients with ALOC $(\mathrm{OR}=3.4$, CI: $1.2-10)$.

Conclusion:

1) Comparing paramedic assigned transport codes to dispatch levels identifies dispatch error but inflates apparent undertriage. Actual under-triage was $42 / 5,737(0.7 \%)$.

2) Though low priority dispatch posed a potential life/limb threat in 7 cases, no adverse outcomes were encountered. Average high/low priority response times differ by $<2.5 \mathrm{~min}$ in San Diego's single tiered EMS system(HP 7:25/LP 9:52); undertriage consequences may differ in other EMS models.

3) Dispatcher protocol deviations, secondhand information, particularly from law enforcement, and cases involving alcohol or ALOC were associated with under-triage in this sample population.
23.

\section{Ambulance Sphygmomanometer Accuracy: Can It Affect Triage to the Trauma Center?} Charles E. Cady, Junior Med Student, Ronald G. Pirrallo, MD, MHSA, Clarence E. Grim, $M D$

Department of Emergency Medicine, Medical College of Wisconsin, Milwaukee, Wisconsin USA

Objectives: To determine the accuracy of sphygmomanometers (SPHYGs) from a metropolitan EMS system and quantitate the mis-triage of adult blunt trauma patients based on erroneous systolic blood pressure (SBP) readings.

Methods-A: A cross-sectional, convenient sample of 150 SPHYGs was checked for accuracy using industry standards. Mean high and low deviations were calculated at $90 \mathrm{mmHg}$.

Methods-B: Retrospectively, a frequency distribution of the initial SBPs of all blunt trauma patients, age $\geq 21$, seen in 1994 was plotted to characterize our study population. The numbers of patients potentially over- or under-triaged were identified when their reported SBP was corrected for using the mean high and low deviation plus 2 SDs.

Results-A: Overall, $25.3 \%$ of the SPHYGs were inaccurate. At $90 \mathrm{mmHg}, 28.0 \%(42 / 150)$ were inaccurate with $16.7 \%(7 / 42)$ high by $4.6 \pm 1.5 \mathrm{mmHg}$ and $81.0 \%(34 / 42)$ low by $6.2 \pm 4.2$ $\mathrm{mmHg}$; one was inoperable.

Results-B: 1,005 adult blunt trauma patients were evaluated; 61 were eliminated: 35 had initial SBPs of $0 \mathrm{mmHg}$ and 26 had no SBP recorded $(n=944)$. The mean initial SBP was $138 \pm 30$ $\mathrm{mmHg}$, and $3.8 \%(36 / 944)$ of the patients had SBPs $<90$ mmHg. Potentially, 2.0\% (19/944) of the patients were undertriaged (initial erroneously high SBP reading $90-98 \mathrm{mmHg}$ ) and $2.5 \%$ (24/944) over-triaged (initial erroneously low SBP reading $74-90 \mathrm{mmHg}$ ).

Conclusions: This study suggests that an accurate SBP measurement at $90 \mathrm{mmHg}$ may not be reliably obtained with $28 \%$ of the sphygmomanometers currently used in our EMS system. Erroneous initial SBP readings due to inaccurate sphygmomanometers alone may mis-triage up to $4.5 \%$ of adult blunt trauma patients. 